\title{
Handgrip strength is associated with suicidal thoughts in men: Cross-sectional analyses from NHANES
}

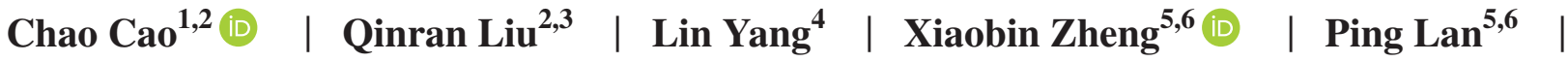 \\ Ai Koyanagi $^{7,8}$ | Davy Vancampfort ${ }^{9,10}$ | Pinar Soysal $^{11}$ | Nicola Veronese ${ }^{12}$ | \\ Brendon Stubbs $^{13,14,15}$ ｜ Joseph Firth ${ }^{16,17}$ | Lee Smith ${ }^{18}$ (D) \\ ${ }^{1}$ Program in Physical Therapy, Washington University School of Medicine, St Louis, MO, USA \\ ${ }^{2}$ Division of Public Health Sciences, Department of Surgery, Washington University School of Medicine, St Louis, MO, USA \\ ${ }^{3}$ Brown School, Washington University in St. Louis, St Louis, MO, USA \\ ${ }^{4}$ Department of Epidemiology, Medical University of Vienna, Vienna, Austria \\ ${ }^{5}$ Department of Colorectal Surgery, the Sixth Affiliated Hospital, Sun Yat-sen University, Guangzhou, China \\ ${ }^{6}$ Guangdong Provincial Key Laboratory of Colorectal and Pelvic Floor Diseases, the Sixth Affiliated Hospital, Sun Yat-sen University, Guangzhou, China \\ ${ }^{7}$ Research and Development Unit, Parc Sanitari Sant Joan de Déu, Universitat de Barcelona, Fundació Sant Joan de Déu, Barcelona, Spain \\ ${ }^{8}$ Centro de Investigación Biomédica en Red de Salud Mental, Instituto de Salud Carlos III, CIBERSAM, Madrid, Spain \\ ${ }^{9}$ Department of Rehabilitation Sciences, KU Leuven, Leuven, Belgium \\ ${ }^{10}$ KU Leuven, University Psychiatric Centre KU Leuven, Leuven-Kortenberg, Belgium \\ ${ }^{11}$ Geriatric Center, Kayseri Education and Research Hospital, Kayseri, Turkey \\ ${ }^{12}$ National Research Council, Aging Branch, Neuroscience Institute, Padova, Italy \\ ${ }^{13}$ Physiotherapy Department, South London and Maudsley NHS Foundation Trust, London, UK \\ ${ }^{14}$ Health Service and Population Research Department Institute of Psychiatry, Psychology and Neuroscience, King's College London, London, UK \\ ${ }^{15}$ Faculty of Health, Social Care and Education, Anglia Ruskin University, Chelmsford, UK \\ ${ }^{16}$ School of Science and Health, NICM Health Research Institute, University of Western Sydney, Sydney, NSW, Australia \\ ${ }^{17}$ Division of Psychology and Mental Health, University of Manchester, Manchester, UK \\ ${ }^{18}$ The Cambridge Centre for Sport and Exercise Sciences, Anglia Ruskin University, Cambridge, UK
}

\section{Correspondence}

Ping Lan and Xiaobin Zheng, the Sixth Affiliated Hospital, Sun Yat-sen University, 26 Yuancun Erheng Road, Guangzhou, Guangdong 510655, China.

Emails: lanping@mail.sysu.edu.cn;

zhengxbn@mail3.sysu.edu.cn

\section{Funding information}

China Postdoctoral Science Foundation,

Grant/Award Number: 2019TQ0390
Objective: To investigate the association between handgrip strength and suicidal thoughts in a representative sample of the US adult population using data from the National Health and Nutrition Examination Survey (NHANES).

Population and Methods: Data from two waves of NHANES (2011-2014) were aggregated. Handgrip strength in kilogram $(\mathrm{kg})$ was defined as the maximum value from the dominant hand. Suicidal thoughts were assessed using one question "Over the last 2 weeks, how often have you been bothered by the following problem: Thoughts that you would be better off dead or of hurting yourself in some way?" and dichotomized to no (not at all) and yes (several days/ more than half the days/ nearly every day). Sex-specific logistic regressions were carried out to analyze associations between handgrip strength and suicidal thoughts. 
Results: Data on total of 8903 adults (mean age $47.4 \pm 0.4$ years) were analyzed. Each $5 \mathrm{~kg}$ increase in handgrip strength was associated with a $16 \%$ reduced odds of having suicidal thoughts $(0.84,95 \% \mathrm{CI}$ : $0.74-0.95)$ among the overall male population. These associations were stronger in male aged $20-39$ years $(0.83,95 \% \mathrm{CI}$ : 0.70-0.98), and 40-64 years (0.73, 95\% CI: 0.63-0.85). In contrast, no associations were observed in females of all age groups.

Conclusions: Males younger than 65 years old with low handgrip strength are significantly more likely to have suicidal thoughts demonstrating a dose-response relationship. Future research is required to confirm/refute our findings and establish if strength interventions can reduce suicidal thoughts.

\section{K E Y W O R D S}

handgrip strength, mental health, physical activity, suicidal thought

\section{$1 \mid$ INTRODUCTION}

More than 800000 people die by suicide each year, and there may have been more than 20 others attempting suicide for each completed suicide. ${ }^{1}$ The US suicide mortality rate in 2016 was 15.3 per 100000 , well above the global average (10.6 per 100000$).{ }^{2}$ Consequently, suicide prevention is an important part of the Mental Health Action Plan of the World Health Organization (WHO), with the goal of reducing the rate of suicide in each country by $10 \%$ between 2012 and $2020 .^{3}$ A precursor to suicide per se is suicidal thoughts. It is estimated that the cross-national lifetime prevalence (standard error) of suicidal ideation is $9.2 \%$, and of these, $60 \%$ transition to plan and attempt within the first year after ideation onset. ${ }^{4}$

Having a mental health condition has been shown to be an important risk factor for suicide ideation..$^{5-7}$ Emerging research suggests handgrip strength is associated with mental health complications in adults of both sexes. ${ }^{8}$ Literature shows weaker handgrip strength to be associated with a lower physical quality of life, ${ }^{9-11}$ which in turn, is likely to have a detrimental impact on mental health. ${ }^{12,13}$ On the other hand, those with mental health complications might be more likely to develop weaker handgrip strength, in so far as mental health complications might cause a decline in systemic physical functioning. ${ }^{14}$ It has been suggested that handgrip strength could be used as a simple tool in clinic to identify those at risk of or suffering from mental health complications as well as disability. ${ }^{15}$

Suicidal ideation is not classified as a mental disorder per se but is indeed a trait of many mental health complications. ${ }^{5-7}$ It is therefore plausible that handgrip strength is associated with suicidal thoughts. In the only study of its kind to date in one million adolescent males, using the Swedish military conscription register, it was found that higher muscular strength (highest 10th vs lowest 10th knee extension and handgrip) was associated with a 20\%-30\% lower risk of death from suicide. ${ }^{16}$ Similar analyses need to be carried out in other populations of both males and females to investigate if such associations exist to inform public health policy and clinical practice. Importantly, the literature reports a higher prevalence of suicidal ideation in females compared with males. ${ }^{4,17-20}$ Hence, the aim of the present study was to investigate sex-specific association between handgrip strength and suicidal thoughts in a representative sample of the US adult population.

\section{$2 \mid$ METHODS}

\section{1 | Study population}

The National Health and Nutrition Examination Survey (NHANES) is a repeated cross-sectional nationally representative health examination survey study in the United States. The design of the study has been detailed elsewhere. ${ }^{21}$ In brief, the NHANES program collected data using complex, stratified, multistage, clustered samples of civilian, non-institutionalized populations of around 5000 individuals in each survey cycle ( 2 years) that are representative of the US population ages 2 months and older. A mobile examination center (MEC) was used to conduct the physical examination for survey participants. Participants provided written informed consents, and study procedures were approved by the National Center for Health Statistic's research ethics review board. ${ }^{22}$ We extracted and aggregated data on suicidal thoughts, handgrip strength, and other characteristics from NHANES cycles 2011-2012, and 2013-2014. The present sample was restricted to adults aged 20 years or older.

\section{2 | Variables}

\subsection{1 | Suicidal thoughts (outcome variable)}

Suicidal thoughts were assessed using one question "Over the last 2 weeks, how often have you been bothered by the 
following problem: Thoughts that you would be better off dead or of hurting yourself in some way?" (response options: not at all/ several days/ more than half the days/ nearly every day). ${ }^{23}$ Those who responded "Several days," "More than half the days," or "Nearly every day" were classed as having suicidal thoughts. Those who responded "Not at all" were classed as "no suicidal thoughts." 24

\subsection{2 | Handgrip strength (exposure variable)}

The handgrip strength protocol was detailed in the NHANES Muscle Strength Procedures Manual. ${ }^{25}$ In brief, handgrip strength in kilogram $(\mathrm{kg})$ was measured with the Takei Digital Grip Strength Dynamometer over three trials separately by 60 seconds and alternating hands, by a trained research assistant. The present analyses used maximum value of the dominant hand as the exposure variable. ${ }^{26}$ Combined grip strength (the sum of the largest reading from each hand) or average values were further included in sensitivity analyses.

\subsection{3 | Medical conditions}

We extracted information on four chronic conditions, assessed in NHANES, known to be related to suicidal ideation and suicide behaviors: diabetes, cancer, arthritis, and cardiovascular diseases. ${ }^{27}$ Participants were considered as having the chronic disease if they self-reported ever been told by a physician that they have the following conditions: diabetes, cancer, arthritis, congestive heart failure, coronary heart diseases, heart attack, or stroke.

\subsection{4 | Sociodemographic characteristics and self-reported leisure-time physical activity (LTPA)}

Sociodemographic variables include age, sex, race/ethnicity (non-Hispanic White, non-Hispanic Black, Hispanic, or Other), weight, height, education, marital status, and smoking status. ${ }^{28}$ Participants were classified into one of the three age groups: 20-39, 40-64, and 65 years or older. Weight and height were measured by trained technicians using standardized equipment at the time of physical examination in the MEC. Body mass index (BMI) was defined as weight in $\mathrm{kg} /$ (height in meters) ${ }^{2}$ and categorized into standard categories: underweight $\left(<18.5 \mathrm{~kg} / \mathrm{m}^{2}\right)$, normal weight $(18.5$ to $<25 \mathrm{~kg} /$ $\mathrm{m}^{2}$ ), overweight $\left(25\right.$ to $\left.<30 \mathrm{~kg} / \mathrm{m}^{2}\right)$, and obese $(\geq 30.0 \mathrm{~kg} /$ $\left.\mathrm{m}^{2}\right){ }^{29}$ We excluded participants with underweight status owing to potential underlying physical conditions. Education level was divided into four groups: less than high school, high school, some college, and college graduate or above. Marital status was classified into two groups: live with someone (married, and living with partner) and live alone (widowed, divorced, separated, never married). Finally, smoking status was defined by the following: never (did not smoke 100 cigarettes in life and do not smoke now), former smoker (smoked 100 cigarettes in life and do not smoke now), and current smoker (smoked 100 cigarettes in life and smoke now).

The present analyses further included information on physical activity patterns as it has been previously shown to be associated with suicidal ideation. ${ }^{30}$ Physical activity was self-reported by participants using questions based on the Global Physical Activity Questionnaire (GPAQ). Levels of leisure-time physical activity (LTPA) were calculated as the minutes per week that participants reported participating in moderate-to-vigorous-intensity physical activity. Participants reported the frequency, and duration of physical activity in a typical week, at vigorous and moderate intensities, respectively. We summarized the total number of minutes for physical activity in each intensity, where the time spent in vigorous-intensity physical activity was doubled and added to the number of minutes of moderate-intensity physical activity to approximate the metabolic equivalent of task value. ${ }^{31}$

\section{3 | Statistical analyses}

To present nationally representative estimates, survey analysis procedures were used to account for the sample weights (MEC exam weight), stratification, and clustering of the complex sampling design. NHANES adults aged 20 years and older with complete information on suicidal thoughts, handgrip strength, and other characteristics were included in the analyses. We analyzed the descriptive characteristics separately in male and female, due to the documented age-related sex difference in suicidal ideation. ${ }^{32,33}$ Weighted means and standard errors were calculated and presented for continuous variables, while weighted proportions were summarized for categorical variables.

Sex-specific logistic regressions were carried out to analyze the relationships between handgrip strength and suicidal thoughts within each age group. Multivariable models were adjusted for age, race, BMI, education level, marital status, smoking status, level of LTPA, and chronic conditions.

We also conducted the following sensitivity analyses: (a) including the underweight participants; (b) using handgrip strength defined as the combined grip strength (the sum of the largest reading from each hand) of both hands; (c) using handgrip strength defined as the maximum value achieved using either hand. Stratified models using survey analysis procedure were carried out within subpopulations defined within each stratum. All statistical significance was determined at $P<.05$. All statistical analyses were performed using STATA version 14.0 (STATA Corp). All the statistical analyses were performed in 2018. 


\section{3 | RESULTS}

The present study included total 8903 adults aged 20 years or older in the two NHANES waves who have completed data on suicidal thoughts, handgrip strength, and other variables. Participant's mean age was $47.4 \pm 0.4$ years at the time of examination, with mean BMI of $29.2 \pm 0.2 \mathrm{~kg} / \mathrm{m}^{2}$. Significant sex differences were observed in all characteristics (Table 1). For example, there were more males than females who had a higher level of education, lived with someone, was a former or current smoker, had no chronic conditions, were sufficiently active, had higher handgrip strength, and did not have suicidal thoughts (all $P$-values $<.001$ ).

Table 2 summarizes analyses stratified by age groups, for males and females respectively (See overall estimates at Table S1). In the sex-specific adjusted models, a handgrip strength increases by $5 \mathrm{~kg}$ was associated with a $16 \%$ reduced odds of having suicidal thoughts 0.84 (0.74-0.95) among the overall male population. No such association was observed in females.

Associations of handgrip strength with suicidal thoughts differed by age and sex stratum. In males, significant associations between handgrip strength and suicidal thoughts were observed in those aged 20-39 years (OR 0.83, 95\% CI: $0.70-0.98)$ and $40-64$ years $(0.73,95 \%$ CI: $0.63-0.85)$. However, this association was not observed among males aged 65 years or older (0.93, 95\% CI: 0.62-1.39). In contrast, in adjusted models no associations between handgrip strength and suicidal thoughts were observed in females $(0.92,95 \%$ CI: $0.80-1.07)$, nor by each age group: $20-39$ years $(1.15$, 95\% CI: 0.81-1.62), $40-64$ years (0.88, 95\% CI: 0.72-1.07), and 65 years or older $(0.75,95 \%$ CI: $0.52-1.10)$.

Our findings were similar when including the underweight group, using handgrip strength defined as combined grip strength or maximum value achieved using either hand in the sensitivity analyses (data not reported).

\section{4 | DISCUSSION}

Findings from the present study in a large representative sample of the US adult population showed that as handgrip strength increased by $5.00 \mathrm{~kg}$, odds of having suicidal thoughts reduced by $16 \%$ in males. In males, significant associations between handgrip strength and suicidal thoughts were observed across all age groups except for those 65 years and older. In contrast, there were no associations in the overall and any age group for females.

Findings from the present study support that of previous research that has shown that handgrip strength is associated with multiple mental health outcomes. ${ }^{8,34}$ A growing body of literature shows weaker handgrip strength to be associated with a lower physical quality of life, ${ }^{9-11}$ which in turn, is likely to stimulate suicidal thoughts. Indeed, previous studies have shown an association between suicidal ideation and poor quality of life in the adult population. ${ }^{35,36}$ On the other hand, those having suicidal thoughts might be likely to develop weaker handgrip strength, suicidal ideation may result in a decline in systemic physical functioning. For example, one previous study found that higher muscular strength (highest 10th vs lowest 10th knee extension and handgrip) was associated with a 20\%-30\% lower risk of death from suicide in Swedish male adolescents. ${ }^{16}$ However, the association is likely to be owing to a combination of both reduced quality of life and declined systemic physical functioning.

A further recent population-scale study has demonstrated that strong handgrip strength is also positively related to purpose in life, which could also contribute toward explaining the relationships shown here. ${ }^{38}$ Another possible explanation to our findings is the proinflammatory state of participants who experience sarcopenia (loss of muscle mass and muscle strength ${ }^{39}$ may operate the pathway to suicidal thought. Mechanistic studies on suicide have been largely focused on psychosocial factors. The neurobiological basis of suicide and suicidal behavior is less studied. ${ }^{40}$ Nevertheless, abnormal levels of cytokines have been found in serum and profrontal cortex of suicide victims, suggesting that a possible driver of suicidal behavior might be imbalanced cytokines at a proinflammatory state.

It is interesting that in the present study an association between handgrip strength and suicidal thoughts was found only in males whereas the prevalence of suicidal ideation is greater in females than males. ${ }^{4,17-19}$ One plausible explanation for the finding in males only is that handgrip strength is a good indicator for overall strength when measured in standardized conditions ${ }^{41}$ and greater muscle mass generally predicts greater strength; in males, muscularity has been shown to be associated with higher levels of self-esteem than in females. ${ }^{42}$ Those with lower levels of selfesteem are more likely to have suicidal thoughts. ${ }^{43,44}$ Lee found that poor relative handgrip strength defined by handgrip strength/BMI ratio was associated with an increased chance of suicidal thoughts among Korean women. ${ }^{45}$ However, due to the paradox of obesity measured by BMI in the United States, the handgrip strength would be a good indicator of muscular and nutritional status among the US population. ${ }^{46}$ Also, the potential racial disparity may explain the difference because of related cultural and sociodemographic diversities ${ }^{47}$ In males 65 years and older, no association was found between handgrip strength and suicidal thoughts. This finding may be owing to a smaller sample size within this age category compared with others or a small variation in grip strength. Moreover, it may be that this age group is less concerned about muscularity per se. A further consideration is that there may be an effect of 
TA B L E 1 Sociodemographic characteristics of adults aged 20 years and older from the NHANES 2011-2014, by sex

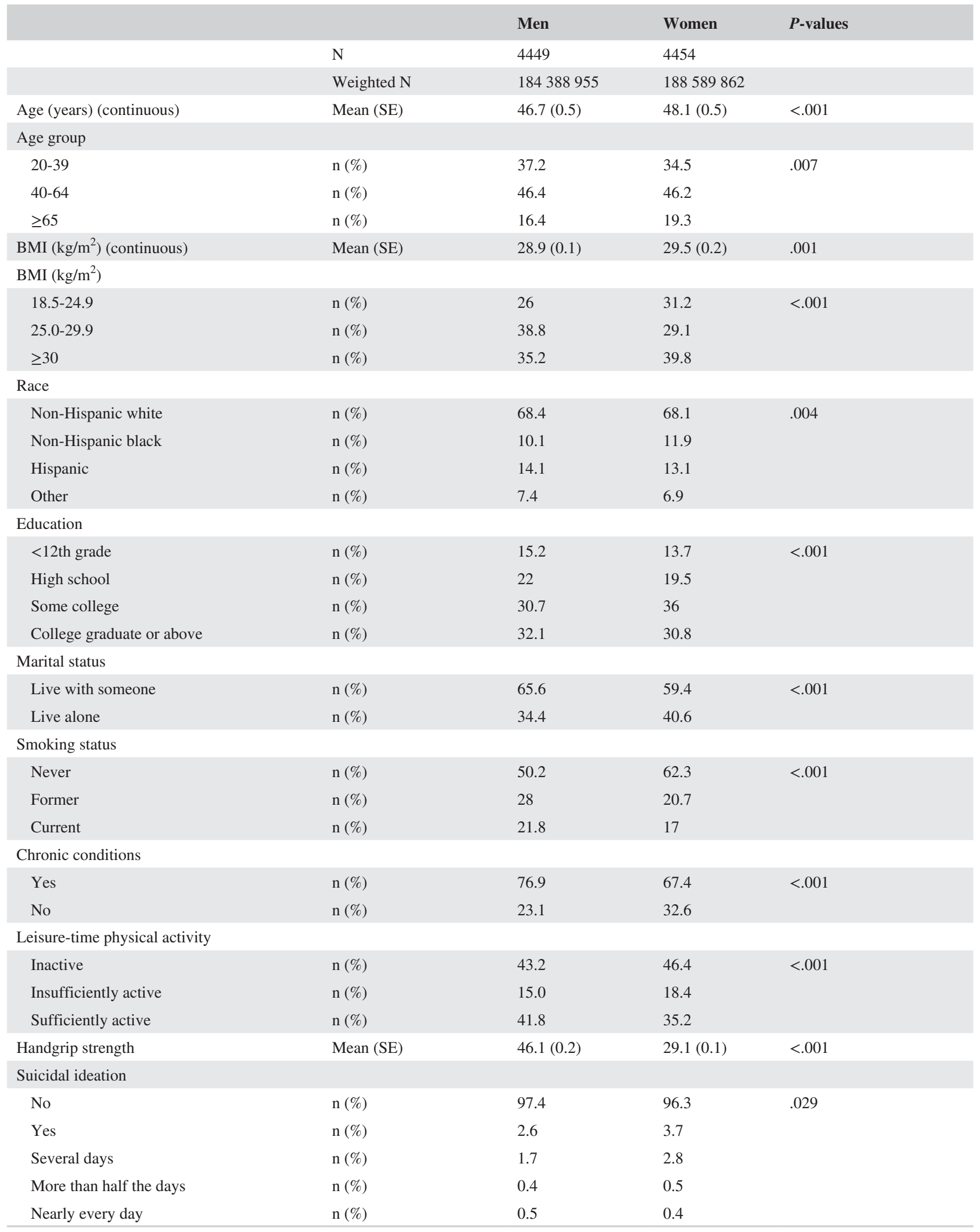




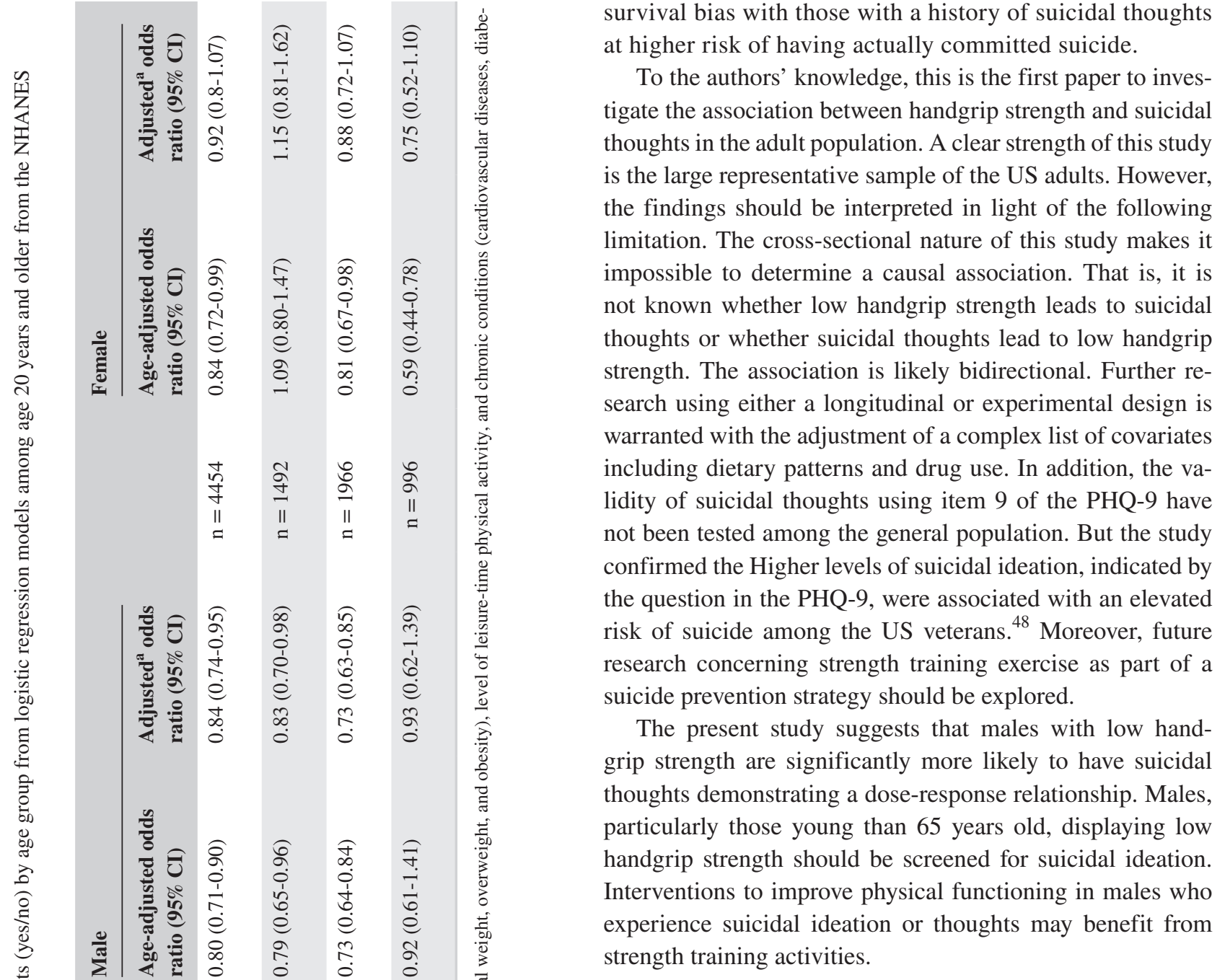

\section{5 | PERSPECTIVES}

The present findings indicate that males younger than 65 years old with low handgrip strength are significantly more likely to have suicidal thoughts, which demonstrates a dose-response relationship. To the best of our knowledge, this is the first assessment on the relationship between handgrip strength and suicidal ideation leveraging US national health data with large representative sample. Consequently, monitoring handgrip strength may be important to identify men with suicidal ideation or thoughts. Given the high suicide mortality rate in the United States, ${ }^{2}$ this finding would be novel in demonstrating handgrip strength as a potential tool for identifying individuals with suicidal ideation. Our study also provides novel strategies which may help prevent suicidal ideation in males by improving physical functioning through strength training activities. We did not observe any associations between handgrip strength and suicidal ideation in the overall and any age group for females. The difference in the sex-specific effect still requires further prospective studies. It is also necessary to examine the mechanisms underlying the relationship of handgrip strength 
with suicidal ideation, independent of depressive mood and other potential confounding factors.

\section{CONFLICT OF INTEREST}

The authors have no conflicts to disclose.

\section{ORCID}

Chao Cao (D) https://orcid.org/0000-0002-0455-1171

Xiaobin Zheng (D) https://orcid.org/0000-0001-9748-6411

Lee Smith (D) https://orcid.org/0000-0002-5340-9833

\section{REFERENCES}

1. World Health Organization. Preventing suicide: a global imperative. 2014; http://www.who.int/mental_health/suicide-prevention/ world_report_2014/en/. Accessed July 22, 2018.

2. The Lancet Public H. Suicide in the USA: a public health emergency. Lancet Public Health. 2018;3(7):e304.

3. World Health Organization. Comprehensive mental health action plan 2013-2020. 2013; http://www.who.int/mental_health/action_ plan_2013/en/. Accessed July 22, 2018.

4. Nock MK, Borges G, Bromet EJ, et al. Cross-national prevalence and risk factors for suicidal ideation, plans and attempts. $\mathrm{Br} J$ Psychiatry. 2008;192(2):98-105.

5. Herzog DB, Greenwood DN, Dorer DJ, et al. Mortality in eating disorders: a descriptive study. Int J Eat Disord. 2000;28(1):20-26.

6. Hovey JD, King CA. Acculturative stress, depression, and suicidal ideation among immigrant and second-generation Latino adolescents. J Am Acad Child Adolesc Psychiatry. 1996;35(9):1183-1192.

7. Kandel DB, Raveis VH, Davies M. Suicidal ideation in adolescence: Depression, substance use, and other risk factors. $J$ Youth Adolesc. 1991;20(2):289-309.

8. Smith L, White S, Stubbs B, et al. Depressive symptoms, handgrip strength, and weight status in US older adults. J Affect Disord. 2018;238:305-310.

9. Giampaoli S, Ferrucci L, Cecchi F, et al. Hand-grip strength predicts incident disability in non-disabled older men. Age Ageing. 1999;28(3):283-288.

10. Rantanen T, Avlund K, Suominen H, Schroll M, Frandin K, Pertti E. Muscle strength as a predictor of onset of ADL dependence in people aged 75 years. Aging Clin Exp Res. 2002;14(3 Suppl):10-15.

11. Rantanen T, Guralnik JM, Foley D, et al. Midlife hand grip strength as a predictor of old age disability. JAMA. 1999;281(6):558-560.

12. Callahan CM, Kroenke K, Counsell SR, et al. Treatment of depression improves physical functioning in older adults. J Am Geriatr Soc. 2005;53(3):367-373.

13. Whiteford HA, Ferrari AJ, Degenhardt L, Feigin V, Vos T. Global burden of mental, neurological, and substance use disorders: an analysis from the global burden of disease study 2010. In: Patel V, Chisholm D, Dua T, Laxminarayan R, Medina-Mora ME eds. Mental, Neurological, and Substance Use Disorders: Disease Control Priorities, Third Edition (Volume 4). Washington, DC: The International Bank for Reconstruction and Development / The World Bank(c) 2016 International Bank for Reconstruction and Development/The World Bank; 2016.
14. Penninx BW, Leveille S, Ferrucci L, van Eijk JT, Guralnik JM. Exploring the effect of depression on physical disability: longitudinal evidence from the established populations for epidemiologic studies of the elderly. Am J Public Health. 1999;89(9):1346-1352.

15. Taekema DG, Gussekloo J, Maier AB, Westendorp R, de Craen A. Handgrip strength as a predictor of functional, psychological and social health. A prospective population-based study among the oldest old. Age Ageing. 2010;39(3):331-337.

16. Ortega FB, Silventoinen K, Tynelius P, Rasmussen F. Muscular strength in male adolescents and premature death: cohort study of one million participants. BMJ. 2012;345:e7279.

17. Kessler RC, Borges G, Walters EE. Prevalence of and risk factors for lifetime suicide attempts in the National Comorbidity Survey. Arch Gen Psychiatry. 1999;56(7):617-626.

18. Eaton DK, Lowry R, Brener ND, Galuska DA, Crosby AE. Associations of body mass index and perceived weight with suicide ideation and suicide attempts among US high school students. Arch Pediatr Adolesc Med. 2005;159(6):513-519.

19. Gunnell D, Harbord R, Singleton N, Jenkins R, Lewis G. Factors influencing the development and amelioration of suicidal thoughts in the general population. Cohort study. Br J Psychiatry. 2004;185:385-393.

20. Husky MM, Guignard R, Beck F, Michel G. Risk behaviors, suicidal ideation and suicide attempts in a nationally representative French sample. J Affect Disord. 2013;151(3):1059-1065.

21. Centers for Disease Control and Prevention. National Health and Nutrition Examination Survey. http://www.cdc.gov/nchs/nhanes. htm. Accessed June 21, 2016.

22. Yang L, Cao C, Kantor E, et al. Trends in sedentary behavior among US. Population from. 2001-2016;321(16):1587-1597.

23. Pratt LA, Brody DJ. Implications of two-stage depression screening for identifying persons with thoughts of self-harm. Gen Hosp Psychiatry. 2014;36(1):119-123.

24. Giddens JM, Sheehan DV. Is there value in asking the question "Do you think you would be better off dead?" in assessing suicidality? a case study. Innov Clin Neurosci. 2014;11(9-10):182-190.

25. Centers for Disease Control and Prevention. National Health and Nutrition Examination Survey (NHANES) Muscle Strength Procedures Manual. 2011.

26. Steiber N. Strong or weak handgrip? Normative reference values for the german population across the life course stratified by sex, age, and body height. PLoS ONE. 2016;11(10):e0163917.

27. Ferro MA, Rhodes AE, Kimber M, et al. Suicidal behaviour among adolescents and young adults with self-reported chronic illness. Can J Psychiatry. 2017;62(12):845-853.

28. Grabovac I, Smith L, Stefanac S, et al. Health care providers' advice on lifestyle modification in the us population: results from the NHANES 2011-2016. Am J Med. 2019;132(4):489-497.e481.

29. World Health Organization. Obesity: preventing and managing the global epidemic. Report of a WHO consultation. World Health Organ Tech Rep Ser. 2000;894:1-253.

30. Vancampfort D, Hallgren M, Firth J, et al. Physical activity and suicidal ideation: a systematic review and meta-analysis. $J$ Affect Disord. 2018;225:438-448.

31. Zhao G, Li C, Ford ES, et al. Leisure-time aerobic physical activity, muscle-strengthening activity and mortality risks among US adults: the NHANES linked mortality study. Br J Sports Med. 2014;48(3):244-249.

32. Freeman A, Mergl R, Kohls E, et al. A cross-national study on gender differences in suicide intent. BMC Psychiatry. 2017;17(1):234. 
33. Perna FM, Coa K, Troiano RP, et al. Muscular grip strength estimates of the U.S. population from the national health and nutrition examination survey 2011-2012. J Strength Cond Res. 2016;30(3):867-874.

34. Fukumori N, Yamamoto Y, Takegami M, et al. Association between hand-grip strength and depressive symptoms: locomotive syndrome and health outcomes in aizu cohort study (LOHAS). Age Ageing. 2015;44(4):592-598.

35. Ponte C, Almeida V, Fernandes L. Suicidal ideation, depression and quality of life in the elderly: study in a gerontopsychiatric consultation. Span J Psychol. 2014;17:E14.

36. Alves VM, Francisco LC, Belo FM, Leão de-Melo-Neto V, Barros VG, Nardi AE. Evaluation of the quality of life and risk of suicide. Clinics. 2016;71(3):135-139.

37. Min J, Min K. Suicide behaviors and health-related quality of life: results from the korean community health survey of 393,073 adults. J Psychiatry. 2014;18:1000214.

38. Kim ES, Kawachi I, Chen Y, Kubzansky LD. Association between purpose in life and objective measures of physical function in older adults. JAMA Psychiatry. 2017;74(10):1039-1045.

39. Schaap LA, Pluijm SM, Deeg DJ, Visser M. Inflammatory markers and loss of muscle mass (sarcopenia) and strength. Am J Med. 2006;119(6):526.e529-517.

40. Pandey GN. Biological basis of suicide and suicidal behavior Bipolar Disord. 2013;15(5):524-541.

41. Roberts HC, Denison HJ, Martin HJ, et al. A review of the measurement of grip strength in clinical and epidemiological studies: towards a standardised approach. Age Ageing. 2011;40(4):423-429.

42. Grossbard JR, Lee CM, Neighbors C, Larimer ME. Body image concerns and contingent self-esteem in male and female college students. Sex Roles. 2009;60(3-4):198-207.

43. Wild LG, Flisher AJ, Lombard C. Suicidal ideation and attempts in adolescents: associations with depression and six domains of self-esteem. J Adolesc. 2004;27(6):611-624.
44. Mitsui N, Asakura S, Shimizu Y, et al. The association between suicide risk and self-esteem in Japanese university students with major depressive episodes of major depressive disorder. Neuropsychiatr Dis Treat. 2014;10:811-816.

45. Lee K. Relative handgrip strength in relation to depressive mood and suicidal ideation in Koreans using the 2015 KNHANES data. $J$ Musculoskelet Neuronal Interact. 2018;18(3):333-338.

46. Norman K, Stobäus N, Gonzalez MC, Schulzke J-D, Pirlich M. Hand grip strength: Outcome predictor and marker of nutritional status. Clin Nutr. 2011;30(2):135-142.

47. Davidson CL, Wingate LR. Racial Disparities in Risk and Protective Factors for Suicide. Journal of Black Psychology. 2011;37(4):499-516.

48. Louzon SA, Bossarte R, McCarthy JF, Katz IR. Does suicidal ideation as measured by the PHQ-9 predict suicide among VA patients? Psychiatric Services. 2016;67(5):517-522.

\section{SUPPORTING INFORMATION}

Additional supporting information may be found online in the Supporting Information section at the end of the article.

How to cite this article: Cao C, Liu Q, Yang L, et al. Handgrip strength is associated with suicidal thoughts in men: Cross-sectional analyses from NHANES.

Scand J Med Sci Sports. 2019;00:1-8. https://doi. org/10.1111/sms.13559 